
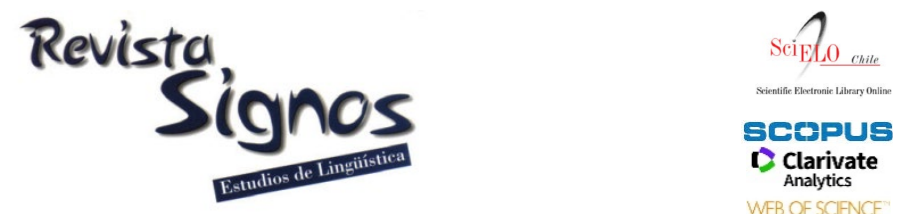

\title{
La perífrasis aspectual 'estar' + gerundio en español infantil. Entre el conocimiento previo y la influencia del input
}

\section{The aspectual periphrasis 'estar' + gerund in Child Spanish. In between previous knowledge and the influence of the input}

\author{
Mary R. Espinosa Ochoa \\ Universidad NACIONAL AutónOMA DE MÉxico \\ MÉXICO \\ maryespinosa@comunidad.unam.mx
}

Recibido: 01-IX-2017 / Aceptado: 26-XII-2018

DOI: $10.4067 /$ S0718-09342019000300759

\section{Resumen}

En este estudio se analiza la adquisición de la construcción perifrástica aspectual 'estar' + gerundio mediante el análisis de una base de datos longitudinal. En la literatura se evidencia que la perífrasis aparece relativamente tarde en la adquisición (JacksonMaldonado \& Maldonado, 2002) y sin una evolución gradual en el desarrollo (Ponce, 2008). En este trabajo se ponen a prueba las siguientes hipótesis: 1) la frecuencia de uso de la perífrasis en el Habla Dirigida al Niño influye en la adquisición y 2) su adquisición es tardía pero repentina porque está apoyada por las construcciones locativas con 'estar' que la preceden en el proceso de adquisición. Para comprobar la primera hipótesis medimos tanto la frecuencia de las ocurrencias como la frecuencia acumulada de tipos de la perífrasis de los niños con respecto al input proveniente de su(s) principal(es) cuidador(es). Para comprobar la segunda hipótesis aplicamos el método de regresión lineal y exponencial utilizando la frecuencia acumulada de tipos de cada una de las construcciones con 'estar'. La frecuencia de las ocurrencias correlaciona con el Habla Dirigida al Niño solo con los datos densos. Sin embargo, la frecuencia acumulada de tipos correlaciona incluso con los datos mensuales. Por otro lado, se observa que las construcciones locativas apoyan la adquisición repentina de la perífrasis.

Palabras Clave: Adquisición de lengua materna, español, Habla Dirigida al Niño, efectos de la frecuencia, perífrasis verbal.

\section{Abstract}

In this study, we undertake a corpus-based analysis of the verbal periphrasis construction estar 'be' + gerund in Spanish. In the literature, there is evidence to support that this periphrasis is relatively late in acquisition Jackson-Maldonado \& 
Maldonado, 2002) and that it appears rapidly, with no apparent gradual development (Ponce, 2008). We tested the following hypotheses: 1) The acquisition of this periphrasis is influenced by Child Directed Speech frequency; 2) The acquisition of the verbal estar 'be' periphrasis is relatively late but seemingly sudden since it is supported by already acquired locative and adjectival estar 'be' constructions. To test the first hypothesis we measured both token and cumulative type frequency of use of the estar 'be' + gerund verbal periphrases of all three caretakers against the children's periphrases, using a Pearson correlation analysis. To test the second hypothesis we applied a lineal and exponential regression method, making use of the cumulative type frequency of each estar 'be' construction. Type frequency is strongly correlated only when Child Directed Speech is correlated with dense data. However, the children's periphrases are strongly correlated with the Child Directed Speech when using cumulative type frequency (once per month). On the other hand, the prior locative construction seems to support the acquisition of this aspectual periphrasis.

Key Words: Spanish, L1 acquisition, child directed speech, frequency effects, verbal periphrasis.

\section{INTRODUCCIÓN}

La adquisición de la perífrasis aspectual con gerundio del español, no ha sido aún objeto principal de ningún estudio específico. Sin embargo, se sabe acerca de su adquisición por medio de otros trabajos que se han interesado por la adquisición del aspecto verbal, la adquisición del verbo 'estar' (y 'ser') y la morfología verbal. Dentro de esta, se ha considerado también la correlación de la morfología con respecto al Habla Dirigida al Niño (en adelante HDN).

Alrededor de los años noventa hubo un auge en el que se puso especial interés en la adquisición infantil del aspecto verbal, debido a que se detectó que existía una congruencia semántica entre el aspecto léxico y el aspecto morfológico (Stoll, 1988; Li \& Bowerman, 1998; Shirai, Slobin \& Weist, 2000; Shirai, 2014). En inglés, por ejemplo, el progresivo -ing (análogo al gerundio en español) es el primero en ser producido por los niños (Brown, 1973). En coherencia con esta congruencia aspectual, los primeros verbos producidos con este morfema progresivo -ing son verbos de actividad. Numerosos estudios han verificado este patrón de adquisición para el inglés (Tomasello, 1992; Behrend, Harris \& Cartwright, 1995).

En español la historia para el gerundio es parcialmente diferente, aunque se confirma la congruencia semántica entre los verbos de actividad y los morfemas imperfectivos (Jackson-Maldonado \& Maldonado, 2002), el gerundio no es el primer morfema en la adquisición. En cambio, el orden en el desarrollo del español como lengua materna se relega hasta el cuarto o quinto lugar (Harrison, 1992; JacksonMaldonado, Maldonado \& Thal, 1998; Mueller-Gathercole, Sebastián \& Soto, 1999) y aparece principalmente dentro de la construcción perifrástica aspectual 'estar' + gerundio que atañe a este trabajo. 
Hernández Pina (1984), quien realizó un estudio de caso sobre la adquisición monolingüe del español, menciona que el verbo 'estar' del niño aparece por primera vez en el vigésimo tercero mes. La investigadora documenta un paralelismo de este verbo con la cópula 'ser'. Así pues, reporta que 'ser' precede a 'estar', el primero aparece en presencia de demostrativos ete(e)s $X$ y después aparece 'estar' para indicar locaciones o estados. Poco después aparece con una frecuencia alta en combinación con gerundios. Este patrón reportado por Hernández Pina (1984), fue también corroborado en el estudio de Ponce (2008), quien hizo un estudio específico sobre la adquisición de las cópulas. Según este estudio, el verbo 'estar' comienza a ser producido en construcciones locativas con función presentativa, principalmente con adverbios locativos, en lo que parecería ser un desarrollo gradual a partir de una construcción congelada por ejemplo 'aquí/stá'. A estas construcciones locativas las suceden construcciones de caracterización que aparecen con adjetivos que tienen rasgos perfectivos, por ejemplo está cerrado, está dormido, está despierto, está mojado. La construcción aspectual, 'estar' +VB-ndo es la última en aparecer y tiene rasgos de productividad sintáctica desde el principio. Esto plantea la primera interrogante de nuestro estudio, ya que la perífrasis aparece en el discurso infantil de una manera repentina y con rasgos de productividad sintáctica, sin que se pueda rastrear un desarrollo paulatino en su adquisición.

Por otro lado, desde una perspectiva emergentista (MacWhinney \& O’Grady, 2015), se ha arrojado evidencia de que el orden de adquisición de cada construcción lingüística corresponde con la frecuencia de las ocurrencias en el HDN (Abbot-Smith $\&$ Behrens, 2006). Además, se ha podido comprobar que el aprendizaje es sensible a la frecuencia lingüística y produce modelamiento neuronal (Romberg \& Saffran, 2011). Este principio se ha comprobado también en cuanto al impacto de la diversidad verbal del input en el desarrollo tanto de lexemas verbales (Hsu, Hadley \& Rispoli, 2017) como de morfemas verbales (Saviciüté, Ambridge \& Pine, 2018). De acuerdo con Mueller-Gathercole et al. (1999), en el caso de los gerundios la historia parecería ser diferente, ya que según sus resultados, los gerundios no están correlacionados con el input.

Mueller-Gathercole et al. (1999) no enfocaron su estudio solamente en esta perífrasis sino que hicieron un estudio general sobre la adquisición de los morfemas verbales del español. Debido a que estas investigadoras argumentan a grosso modo que los gerundios no se correlacionan con el input, decidí volver a comprobar este supuesto sobre la correlación con el input pero utilizando un mayor número de elementos. En primer lugar, aumenté el número de participantes. En vez de utilizar los datos longitudinales de dos niños, utilicé los datos longitudinales de tres niños. En cuanto a la densidad de la muestra, agregué no solo datos recogidos de forma mensual sino una muestra más amplia, la de la niña Flor, que en ciertos periodos de la adquisición fue videograbada dos veces al mes. En segundo lugar, controlé 
ocurrencias (o tokens) y la frecuencia acumulada de tipos, ya que se ha probado que estos últimos pueden ser un buen índice para medir la productividad sintáctica en la adquisición infantil (Ruhland \& Geert, 1998).

El objetivo de este trabajo es identificar los factores que pudieran estar ejerciendo una influencia en la adquisición relativamente tardía pero repentina de la perífrasis aspectual 'estar' + gerundio. Los factores que se analizan son el HDN y el conocimiento previo que el niño ha adquirido en etapas anteriores del proceso de desarrollo lingüístico, con énfasis en las construcciones locativas y adjetivas. Se plantean dos hipótesis en torno a las que gira este estudio:

1) La frecuencia de las perífrasis documentadas en el HDN está correlacionada con el desarrollo de las perífrasis en el habla infantil.

2) Las producciones de la perífrasis aspectual son productivas desde el inicio, debido a que tienen una base semántica en común con las construcciones locativas con 'estar'.

\section{Marco teórico}

\subsection{La propuesta de Abbot-Smith y Behrens}

Una posible respuesta a la adquisición aparentemente repentina de la perífrasis podría encontrarse en la propuesta de Abbot-Smith y Behrens (2006), en la que se plantea que ciertas construcciones aparecen en el discurso infantil como resultado de mecanismos de aprendizaje graduales (Abbot-Smith \& Behrens, 2006; Rojas Nieto, en prensa). Según este acercamiento, ciertas construcciones que comparten elementos similares y que preceden a otras en la adquisición, podrían estar apoyando la adquisición de las sucesoras. Así por ejemplo, Abbot-Smith y Behrens (2006) encontraron que las pasivas del alemán con el verbo sein 'ser/estar' son construcciones apoyadas por otras precedentes en la adquisición que contienen el mismo verbo sein. Este tipo de estudios de corpus se apoya en experimentos recientes que comprueban la importancia de la frecuencia estadística en el aprendizaje, cuyo procedimiento está claramente influenciado por el conocimiento lingüístico previo de los sujetos (Siegelman, Bogaerts, Elazar, Arciuli \& Frost, 2018).

Con base en la aportación de Abbot-Smith y Behrens (2006), se analizaron las descripciones del estudio detallado de Ponce (2008) sobre la adquisición del verbo 'estar' en el español infantil temprano. Este análisis reveló que las construcciones precedentes a la perífrasis aspectual tienen características similares que podrían estar apoyando la adquisición 'repentina' de 'estar' + gerundio. En la Figura 1 se realizó un análisis por etapa de la adquisición del verbo 'estar' basado en Ponce (2008). 
Etapa 1. Dimensión visual-espacial

Ej. Aquí está/No está

Etapa 2. Dimensión espacio-temporal

Ej. Está aquí/allá

Etapa 3. Dimensión de estado

Ej. Está frio (+ perfectivo)

Etapa 4. Dimensión de estadio espacio-temporal

(+ imperfectivo). E.g. Está nadando "It is swimming'.

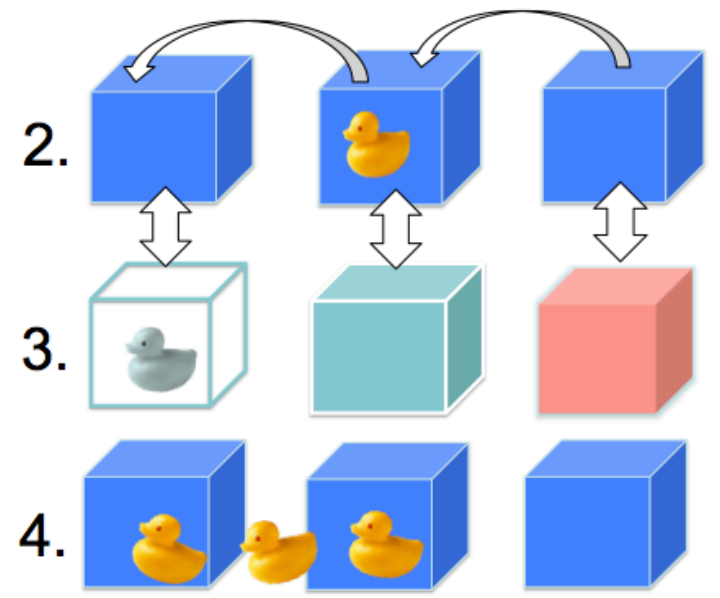

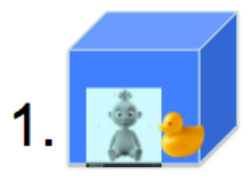

Etapa 1. El verbo estar es producido por el niño para identificar si un objeto es accesible en su campo visual

Etapa 2. De desarrollo gradual: El referente del sujeto en una construcción con estar puede cambiar su locación temporal.

Etapa 3. Generalización analógica. El referente de un sujeto en una construcción con estar puede cambiar su estado temporal.

Etapa 4. Generalización analógica. El referente de un sujeto en una construcción con estar puede experimentar un rápido cambio espacio-temporal.

Figura 1. Análisis cognitivo de las etapas de adquisición temprana del verbo 'estar'.

A continuación se explica con más detalle cada una de las etapas:

En la etapa 1 los niños utilizan el verbo 'estar' para identificar si el referente del sujeto es accesible o inaccesible a la vista, o quizás incluso al tacto (aun cuando estas construcciones 'aquí está'/'no está' son enunciados de base léxica).

Ejemplo 1.

\section{Contexto:}

La niña busca el jabón en la bañera

NIÑA: Aquí está (al momento en que saca el jabón del agua)

En la etapa 2, mediante un desarrollo gradual de base léxica, los niños utilizaron las construcciones locativas con 'estar'. Se propone aquí que en esta etapa se asocia al referente del sujeto de la construcción locativa con 'estar' con un objeto que puede cambiar de locación en el tiempo. En el ejemplo 1, la niña está en la bañera, el jabón se resbala constantemente y, por ende, su locación cambia; durante la hora del baño se emite el verbo 'estar' con adverbios locativos del español como predicativos: '(el jabón) está ahí', 'está allí', 'está aquí'. 
En la tercera etapa los niños podrían establecer una analogía del referente del sujeto del verbo 'estar' como cambiante, ya que el estado del referente del sujeto en una construcción con 'estar' cambia en situaciones propias del ámbito infantil y ante sus propios ojos (y los demás sentidos). En el ejemplo 2 presentamos este escenario del HDN que permite concebir que el referente del sujeto del verbo 'estar' es cambiante.

Ejemplo 2. El referente del sujeto en todas las construcciones con 'estar' es el agua.

\section{Escenario 1.}

Contexto: La niña está por meterse a la bañera. Mete un pie al agua y lo saca inmediatamente.

Cronometraje del video: 00:00:03

MAMÁ: ¿Está caliente?

Escenario 2.

Contexto: La niña no quiere meterse al agua a pesar de la insistencia de la madre.

Cronometraje del video: 00:01:26

NIÑA: ete (=caliente?)

MAMÁ: No está tan caliente mi amor

\section{Escenario 3:}

Contexto: La niña está ahora adentro de la bañera pero parada y no quiere sentarse.

Cronometraje del video: 00:02:54

MAMÁ: Ya no está tan caliente

\section{Escenario 4.}

Contexto: Ahora la madre le pide a la niña que se salga de la bañera, la niña se sale por 5 segundos, pero cuando la madre toma la toalla para ponérsela, la niña se mete de nuevo a la bañera.

Cronometraje del video: 00:24:01

MAMÁ: Ya está fría mi amor

Se trata de un único referente (el agua) que cambia de estado y cuya identificación se expresa en combinación con el verbo 'estar'. En esta tercera etapa se habría fortalecido el significado del verbo 'estar' como asociado con un referente que puede, potencialmente, cambiar de lugar y cambiar de estado. Proponemos que el gerundio 
genera esta imagen: si pensamos en el ejemplo del esquema 'nadando', podemos imaginar una escena de tipo cinematográfica que genera una imagen en movimiento, dinámica, cambiando de lugar en el espacio y el tiempo en una fase del evento 'nadando' determinada. En la Figura 1, en el apartado 4, podríamos situar al pato en un punto $\mathrm{A}$, con la pata izquierda detrás y la pata derecha adelante, en la siguiente imagen estará en un punto B, con la pata izquierda adelante y la pata derecha atrás, etc. En la literatura, el gerundio se ha analizado como un codificador del aspecto de fase progresiva de un estado de cosas dinámico que genera lecturas de caso (Soto \& Castro, 2010). También se ha dicho que el gerundio se combina con 'estar' ya que refiere estadios (Fernández Leborans, 1999). Estas lecturas apoyan nuestro argumento en el sentido de que su lectura de fase/estadio permite establecer una analogía con las construcciones que la preceden en la adquisición. Así que, independientemente de la frecuencia del gerundio en el input, una vez adquirida la construcción locativa, el niño solo necesitaría conocer o dominar el uso de la perífrasis en un contexto específico, con un solo verbo. Este conocimiento le permitiría generalizar su uso a otros contextos, con otros verbos, debido a que domina el uso del verbo 'estar'. Dado que el gerundio genera esta imagen de cambio/fase/estadio, el niño podría establecer una analogía mediante la cual puede omitir el desarrollo gradual de la perífrasis. Consideramos también que la perífrasis de gerundio puede ser organizada por la mente del niño por medio de lo que Goldberg (1995) llama pre-emption, que traducimos como 'derecho preferente' a elegir probabilísticamente una construcción que expresa la misma intención en el mensaje. En este sentido, tanto la construcción locativa 'está + LOC' ejemplo 'está ahí', como la construcción perifrástica en cuestión puede funcionar como respuesta a la pregunta ¿dónde está?, en el que la respuesta puede ser tanto 'está ahí' como 'se está bañando'. Este tipo de respuesta la podemos cotejar con una respuesta infantil del corpus:

(1) Mamá: ¿Dónde está tu tía?

Niña: ta mañano = se está bañando

Otro razonamiento para esta propuesta es que, en esta etapa, el niño ha acumulado un cierto número de verbos en su acervo cognoscitivo que le permiten además formar un patrón para generalizar la raíz verbal más el morfema -ndo del gerundio. Después podría combinarla con 'estar', o concretamente, con 'está', conjugado en tercera persona en presente de indicativo. Es decir, la adquisición de 'está' + gerundio correspondería a una etapa posterior o coincidente con la llamada etapa protomorfológica (Bittner, Dressler \& Kilani-Schoch, 2003) en la que los verbos ya no se producen solo con un morfema específico sino que toma lugar la detección de patrones y sucede una generalización de raíces con nuevos morfemas verbales. Cuando el niño se encuentra en esta etapa, puede detectar que la raíz -ndo es aplicable a verbos y dado su dominio del verbo 'estar', puede establecer una analogía con la 
construcción locativa precedente que lo llevaría a una producción 'repentina' de la perífrasis.

\subsection{Modelo conexionista}

Este modelo propone que la adquisición es un proceso en el que tiene lugar la capacidad de la mente para organizar ciertas estructuras gracias a la frecuencia de las construcciones, la relevancia de la intención comunicativa y las propiedades semánticas de cada construcción individual (Ambridge \& Blything, 2016). Esto explicaría, por ejemplo, la generalización y sobregeneralización que hacen los niños en el proceso de adquisición de las estructuras argumentales. En inglés, estos factores explicarían el uso infantil de una construcción de dativo de doble objeto (p. ej. *Marge pulled Homer the box), en el lugar donde debe ir una construcción de dativo de objeto preposicional (p. ej. Marge pulled the box to Homer (Ambridge \& Blything, 2016). En primera, algunos verbos que aceptan una u otra construcción comparten similitud semántica. En segunda, la construcción de dativo de doble objeto y la de dativo preposicional sirven a propósitos comunicativos similares. En tercera y última instancia, los niños activan cada construcción en proporción a la frecuencia con que cada verbo ha aparecido en esa construcción en las oraciones del input. Los niños se basan en un templete del tipo [VBO.ARRASTRAR CAUSA $_{\text {SUSTANTIVO }}$ AGENTE SUSTANTIVO TEMA + TO SUSTANTIVO DESTINATARIO].

La propuesta conexionista de este estudio consiste en que, para comprender la adquisición de la construcción aspectual, los niños parten de la construcción locativa,

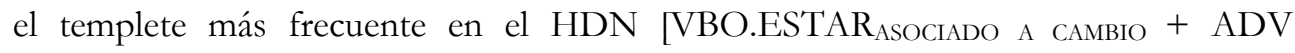
LOCATIVO (CAMBIANTE)]. Los niños insertan en este templete un verbo en gerundio, con propiedades semánticas de fase/estadio, similares a las del adverbio. Tanto la construcción locativa como la aspectual sirven a propósitos comunicativos similares, tal como lo mencionamos arriba, ambas construcciones responden a la pregunta: ¿dónde está? Estas características permiten adscribir esta propuesta a un modelo conexionista.

\subsection{Propuesta de investigación}

En esta investigación pretendemos comprobar si las construcciones locativas y adjetivas con 'estar' previas a la adquisición de la perífrasis aspectual 'estar' + gerundio apoyan la aparición repentina de esta última. Según Abbot-Smith y Behrens (2006) la condición para que una construcción apoye a otra es que compartan material léxico y por tanto similitud semántica. Según expusimos arriba (ver Figura 1), este parece ser el caso para la construcción aspectual, ya que comparte no solo material léxico con las construcciones locativas y adjetivas con 'estar', sino que además se puede ver una relación analógica entre ellas. 


\section{Metodología}

\subsection{Corpus}

Con el fin de llevar a cabo este estudio se utilizó la base de datos longitudinal de ETAL Etapas Tempranas en la Adquisición del Lenguaje (Rojas Nieto, 2007) del Instituto de Investigaciones Filológicas de la Universidad Nacional Autónoma de México. Tres niños son los sujetos de investigación: Elia, Julio y Flor. Los tres son monolingües del español y pertenecen a la clase media escolarizada de la Ciudad de México. Los tres niños fueron videograbados en escenas de la vida cotidiana en interacción espontánea con su(s) principal(es) cuidador(es).

\subsection{Características de los participantes}

Las edades de los tres niños abarcaron el siguiente rango: Elia (1;10-3;08), Julio $(2 ; 05-3 ; 11)$ y Flor $(1 ; 09-4 ; 00)$. Corpus mensual: Elia y Julio fueron videograbados aproximadamente una vez al mes, por lo que los datos analizados de estos dos niños constituyen lo que llamaremos corpus mensual. Aunque de la niña Flor se tiene un número mayor de datos, utilizamos solamente el equivalente a una hora de grabación al mes para integrar los datos de Flor a este corpus. En otras palabras, el mismo número de videograbaciones fueron consideradas para los tres niños, en etapas similares de la adquisición para estructurar el corpus mensual.

Flor fue videograbada en un lapso promedio de 20 días durante cierto periodo de su adquisición, cuando la investigadora observó que la niña pasaba por una etapa de adquisición rápida. Las videograbaciones posteriores a este periodo tomaron lugar una vez al mes. El corpus de Flor es lo que constituye lo que llamaremos 'corpus denso', ya que, aunque abarca el mismo lapso de desarrollo que Elia y Julio, contamos con mayor número de registros.

El HDN fue seleccionado de la siguiente manera: i) Se consideró al principal cuidador de cada niño; ii) el principal cuidador tenía que ser también la persona que más presencia tuviera durante las videograbaciones. Así pues, el HDN seleccionado para su correlación con los niños Flor y Julio fue la madre respectiva de cada uno. En el caso de Elia, se seleccionó tanto el habla de la madre como del padre, ya que ambos interactúan en igual medida con la niña durante las videograbaciones.

\subsection{Procedimiento del análisis}

Para comprobar la primera hipótesis medimos tanto la frecuencia de las ocurrencias como la frecuencia acumulada de tipos. Esta última consiste en contar los gerundios que aparecen con la perífrasis con 'estar' únicamente una vez. Por ejemplo, si una de las madres dice en la primera videograbación 'está jugando' y en la cuarta videograbación la repite, esta emisión no se vuelve a contar. El argumento por el cual 
decidimos proceder de esta manera se debe a que la frecuencia acumulada de tipos es una medida fiable para medir la productividad infantil (Ruhland \& Geert, 1998). Con respecto a utilizarla como medida para el HDN, parecía razonable indagar en qué medida están proveyendo al niño con nuevos ejemplares, ya que podía ser posible que el niño alcanzara un uso productivo de la perífrasis habiéndola escuchado con nuevos gerundios en nuevos contextos.

En el conteo, se descartó las construcciones que aparecían como parte de lectura de libros, canciones y repeticiones inmediatas del discurso precedente, dicho de otro modo, se contaron únicamente las emisiones espontáneas. Una vez recolectados los datos tanto de las ocurrencias como de la frecuencia acumulada de tipos, aplicamos una prueba de correlación Pearson de cada niño con los datos de su(s) cuidador(es) correspondiente(s).

Para comprobar la segunda hipótesis consideramos todas las construcciones con 'estar' emitidas por los niños de manera espontánea y aplicamos un análisis de regresión lineal y exponencial. Este método resulta útil para medir si el crecimiento de una construcción depende de otra y está basado en el análisis estadístico realizado por Abbot-Smith y Behrens (2006). Siguiendo a estas autoras, llevé a cabo un análisis de correlación lineal y exponencial que sirve para indagar en qué medida el uso de una construcción depende de otra y si existe conocimiento previo de una determinada construcción al momento de ser utilizada por primera vez por el niño.

\section{Resultados}

\subsection{Primera hipótesis}

En la primera hipótesis de este trabajo nos preguntamos sobre la relevancia de la influencia del HDN en la adquisición de la perífrasis aspectual. Partiendo del principio de que las ocurrencias de cada construcción predice el orden de adquisición, consideré entonces en primer lugar las ocurrencias del HDN, para comprobar si tienen alguna correlación con la adquisición de la perífrasis infantil. Utilizamos las ocurrencias tanto de la base de datos densa (Flor) como la base de datos mensual para los tres niños. Los resultados fueron reproducidos en la Tabla 1. 
Tabla 1. Correlación de las ocurrencias de la perífrasis del HDN con el habla infantil.

\begin{tabular}{|l|c|c|c|c|c|}
\hline SUJETO & Edades & $\begin{array}{c}\text { Horas de } \\
\text { interacción } \\
\text { estudiadas }\end{array}$ & $\begin{array}{c}\text { Valor de la } \\
\mathbf{R}^{\mathbf{2}}\end{array}$ & $\begin{array}{c}\text { Valor de } \\
\mathbf{l a ~} \mathbf{P} \text { en } \\
\mathbf{p}<\mathbf{0 . 0 5}\end{array}$ & CORRELACIÓN \\
\hline $\begin{array}{l}\text { FLOR } \\
\text { Corpus } \\
\text { Denso }\end{array}$ & $1 ; 10,24-4 ; 00,00$ & 42 & 0.6788 & 0.000717 & $\begin{array}{c}\text { Correlación positiva alta } \\
\text { Significativa }\end{array}$ \\
\hline FLOR & $1 ; 10,24-3 ; 02,00$ & 28 & .378 & 0.182672 & $\begin{array}{c}\text { Correlación positiva } \\
\text { débil. } \\
\text { No significativa. }\end{array}$ \\
\hline ELIA & $1 ; 10,22-3 ; 01,28$ & 28 & -0.3334 & 0.244671 & $\begin{array}{c}\text { Negativa } \\
\text { No significativa. }\end{array}$ \\
\hline JULIO & $2 ; 08-3 ; 11$ & 28 & -0.1387 & 0.638019 & Negativa no significativa \\
\hline
\end{tabular}

Como se puede observar, la frecuencia de las ocurrencias de los niños correlaciona con el input únicamente en la base de datos densa, de hecho, esta correlación en p < 0.05 es positiva alta y significativa. Cuando realizamos el mismo cálculo para la niña Flor y su madre utilizando el mismo número de horas y con el mismo intervalo (mensual) que para Elia y Julio, obtenemos en cambio una correlación positiva pero débil y no significativa. En los casos específicos tanto de Elia y sus padres, como de Julio y su madre, obtenemos una correlación negativa. Esta tabla sugiere que para averiguar la influencia del HDN sobre el desarrollo de cierta construcción debemos tener en cuenta que una base de datos longitudinal mensual no siempre es suficiente para estos propósitos, especialmente cuando la construcción no es muy frecuente en el habla y se restringe a determinados contextos de uso en las actividades de la vida cotidiana de los niños.

Según Ruhland y Geert (1998) la frecuencia acumulada de los primeros 25 tipos del elemento lingüístico estudiado permite dar cuenta de si existe productividad sintáctica ${ }^{1}$ en la adquisición. Siguiendo esta línea, tomé los primeros 25 gerundios que aparecen en la construcción infantil 'estar' + gerundio y los correlacioné con los primeros 25 gerundios que aparecen en el HDN. Se tomó como punto de partida el primer gerundio de los niños, ya que sabemos que los adultos son productivos en su lenguaje, en este caso, en el uso de la perífrasis. Esta correlación se realizó únicamente con el corpus mensual. Los resultados pueden verse en la Tabla 2. 
Tabla 2. Frecuencia acumulada de los primeros 25 tipos de gerundio.

\begin{tabular}{|l|l|l|l|l|l|}
\hline SUJETO & Edades & $\begin{array}{c}\text { Horas de } \\
\text { interacción } \\
\text { estudiadas }\end{array}$ & $\begin{array}{c}\text { El valor } \\
\mathbf{d e ~} \mathbf{l a} \mathbf{R}^{\mathbf{2}}\end{array}$ & $\begin{array}{c}\text { Valor de } \mathbf{P} \\
\mathbf{e n} \\
\mathbf{p}<\mathbf{0 . 0 5}\end{array}$ & \multicolumn{1}{|c|}{ CORRELACIÓN } \\
\hline FLOR & $1 ; 09-2 ; 07$ & 22 & .98 & $<0.00001$ & $\begin{array}{l}\text { Correlación positiva fuerte. } \\
\text { Significativa }\end{array}$ \\
\hline ELIA & $2 ; 03-3 ; 08$ & 30 & .9555 & $<0.00001$ & $\begin{array}{l}\text { Correlación positiva fuerte } \\
\text { Significativa }\end{array}$ \\
\hline JULIO & $2 ; 05-3 ; 07$ & 28 & .7692 & 0.002114 & $\begin{array}{l}\text { Correlación positiva fuerte. } \\
\text { Significativa }\end{array}$ \\
\hline
\end{tabular}

Al realizar la correlación de la frecuencia acumulada de tipos se observa que, a pesar de haber considerado únicamente el corpus mensual, la correlación de la frecuencia acumulada de tipos de gerundio de los niños con respecto al HDN es alta y significativa en los tres casos. Esto permite afirmar que la tasa de crecimiento de los gerundios en las perífrasis producidas por los niños está ligada con los nuevos tipos que los adultos proveen en el HDN. A partir de los resultados de las Tablas 1 y 2 , propongo que las ocurrencias ayudan al niño a identificar la perífrasis en un contexto específico (Tabla 1), pero la productividad sintáctica en el uso dependerá de la frecuencia acumulada de tipos (Tabla 2). Estos datos nos permiten corroborar que en cierta medida y en ciertos momentos de la adquisición de la perífrasis aspectual, el HDN influye de manera significativa en la adquisición, sin embargo, esto no nos permite averiguar por qué la adquisición del gerundio es repentina. Me remito entonces a la comprobación de la segunda hipótesis.

\subsection{Segunda hipótesis}

Para la comprobación de la segunda hipótesis buscamos los primeros 25 tipos de predicativo para cada construcción con 'estar' de cada uno de los niños. De los tres, solamente la niña Elia alcanzó los 25 tipos para cada una de las construcciones. Reproducimos los resultados del análisis lineal y exponencial en la Tabla 3.

Tabla 3. Análisis lineal y exponencial de los primeros 25 de tipos de predicativo de las construcciones con 'estar' de Elia.

\begin{tabular}{|l|c|c|c|}
\hline \multicolumn{1}{|c|}{ ELIA } & Locativos & Adjetivos & Gerundios \\
\hline Lineal & 0.758331 & 0.960192 & 0.98163 \\
\hline Exponencial & 0.943882 & 0.877976 & 0.943882 \\
\hline
\end{tabular}

Siguiendo la línea de análisis de Abbot-Smith y Behrens (2006), una construcción que está siendo aprendida gradualmente del input tendrá un mejor ajuste exponencial; en cambio, una construcción que muestra conocimiento previo desde el principio mostrará un mejor ajuste de crecimiento lineal. El mejor ajuste está dado por la cifra cuyo valor se acerque más a 1 . De acuerdo con esta línea de argumentación solo las construcciones locativas muestran un desarrollo gradual, puesto que alcanzan un 
mejor ajuste exponencial. El desarrollo gradual con mejor ajuste exponencial de las construcciones locativas puede verse representado de forma visual en el Gráfico 1. Este resultado corrobora también el estudio de Ponce (2008) que identifica estas construcciones locativas con 'estar' como de crecimiento gradual de base léxica. Las construcciones con adjetivo y gerundio muestran, en cambio, un mejor ajuste lineal, lo que sugiere que los niños tienen representación semántica de estas construcciones desde el principio. Para los fines específicos de nuestra hipótesis resultaría entonces que la construcción locativa está apoyando la adquisición de la perífrasis aspectual.

Otro aspecto importante en el análisis de correlación es el crecimiento de la curva, una construcción que tiene un desarrollo gradual sin conocimiento previo mostrará también una curva acorde a este desarrollo, es decir, un crecimiento gradual. Este es el caso de las construcciones locativas, cuya curva de crecimiento en la adquisición de la niña Elia vemos representado en el Gráfico 1.

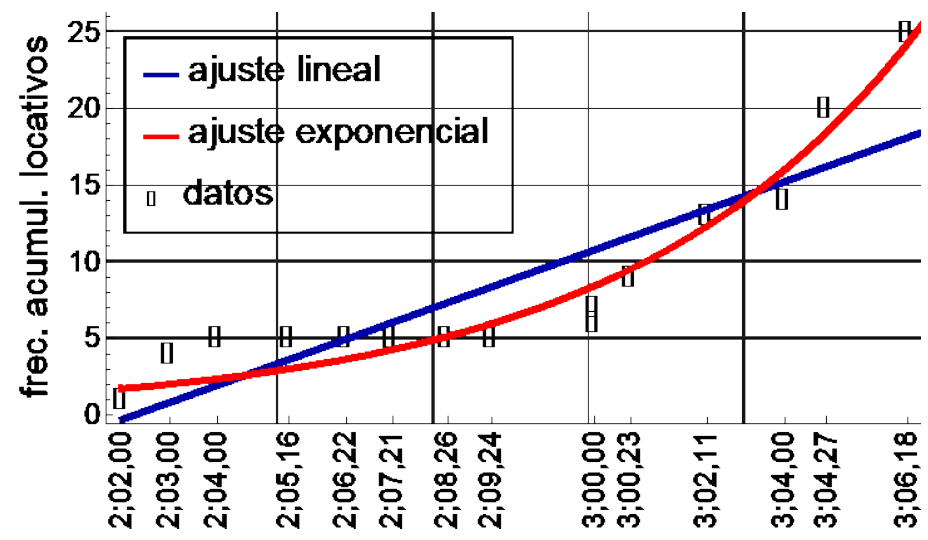

Gráfico 1. Curva de crecimiento gradual de las construcciones locativas de Elia, que muestra un mejor ajuste exponencial.

En cambio, una construcción que crece con apoyo de otras, presentará un uso nulo prolongado por parte del niño, y sus primeros usos mostrarán una curva con pendiente pronunciada. Este es el caso de la construcción que conforma la perífrasis con gerundio y que se puede ver representada en el Gráfico 2. 


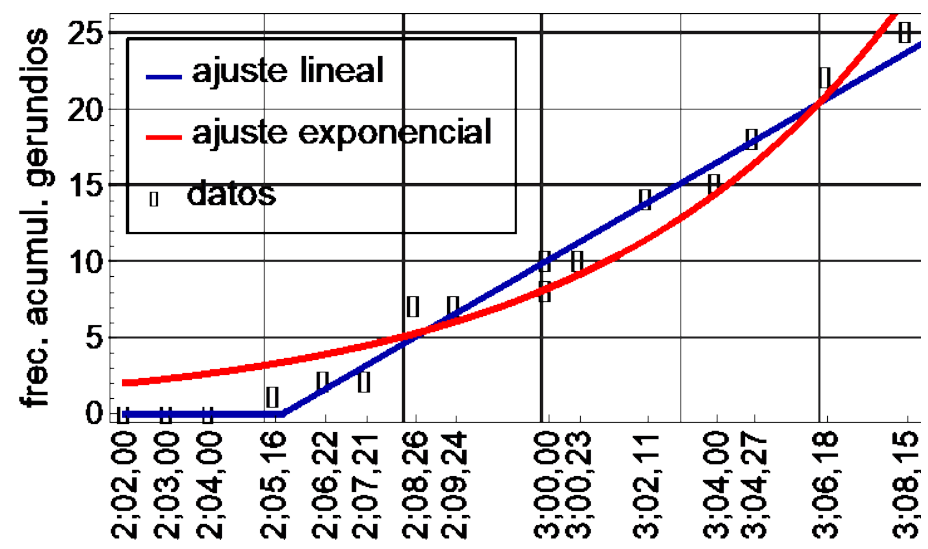

Gráfico 2. Curva de crecimiento de las construcciones con gerundio de Elia que muestra una pendiente pronunciada después de un periodo de aparición nula.

Puesto que Flor y Julio alcanzaron el uso de 25 tipos de gerundios solo en la perífrasis, realizamos el análisis de correlación también para estas construcciones en particular (Tabla 4) y confirmamos que todos los niños muestran conocimiento previo desde el inicio, puesto que muestran un mejor ajuste lineal que exponencial.

Tabla 4. Análisis de correlación para las perífrasis aspectuales de los tres niños.

\begin{tabular}{|l|c|c|}
\hline & Ajuste lineal & Ajuste exponencial \\
\hline Elia & 0.98163 & 0.943882 \\
\hline Flor & 0.985858 & 0.924865 \\
\hline Julio & 0.99022 & 0.957142 \\
\hline
\end{tabular}

A pesar de que los datos de Flor y Julio no acumularon los 25 tipos en las construcciones locativas y adjetivas, y no permiten un análisis de regresión lineal y exponencial, muestro también las gráficas de crecimiento de los predicativos con 'estar' de Flor y Julio. Esto nos da una visión gráfica del momento del surgimiento de la perífrasis aspectual como posterior al de las construcciones locativas.

Como se puede apreciar en los Gráficos 3 y 4, los niños no alcanzaron los 25 tipos en todas las construcciones, sin embargo, nos permite observar que la perífrasis con gerundio surge solamente después de que la niña Flor (Gráfico 3) ya ha estado utilizando las construcciones locativas por un tiempo. 


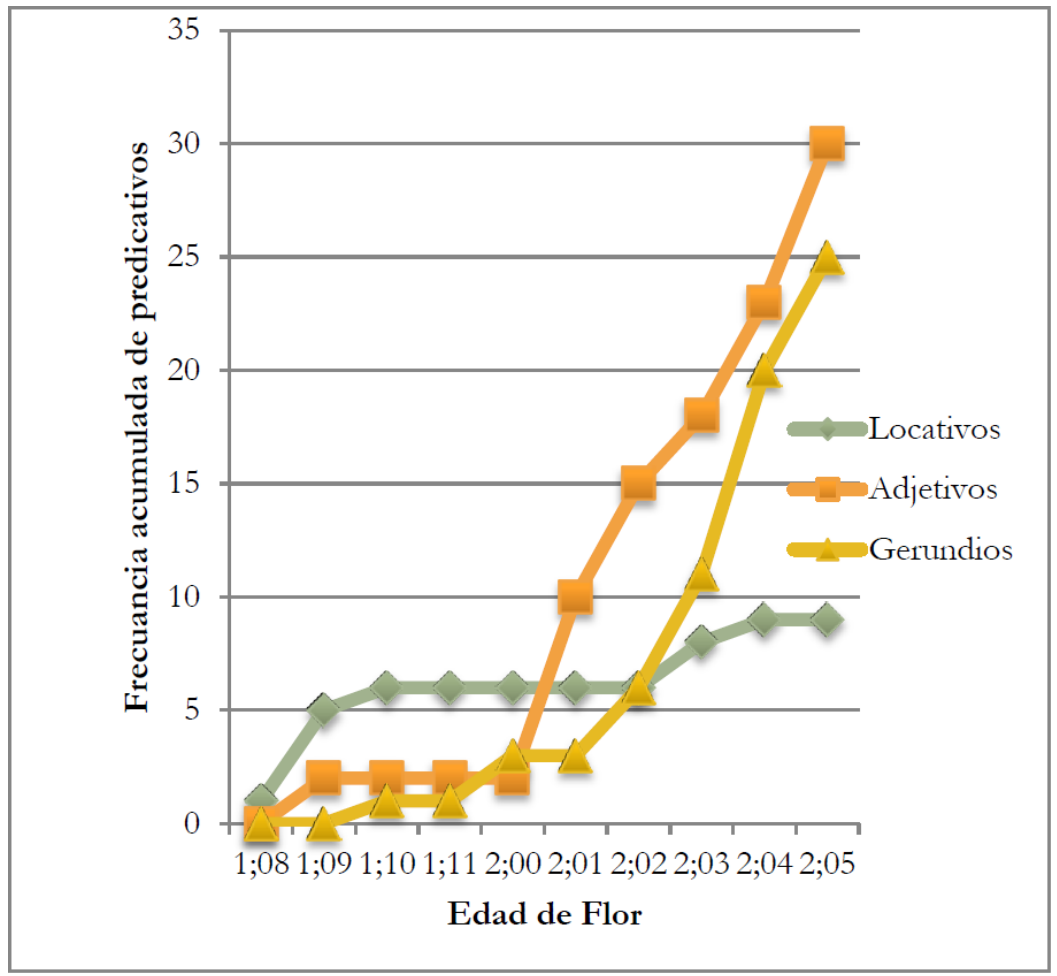

Gráfico 3. Frecuencia acumulada de tipos de predicativo de Flor.

Las construcciones adjetivas no parecen ser parte del apoyo para la construcción aspectual, ya que crecen prácticamente al mismo tiempo y en paralelo con la perífrasis aspectual. 


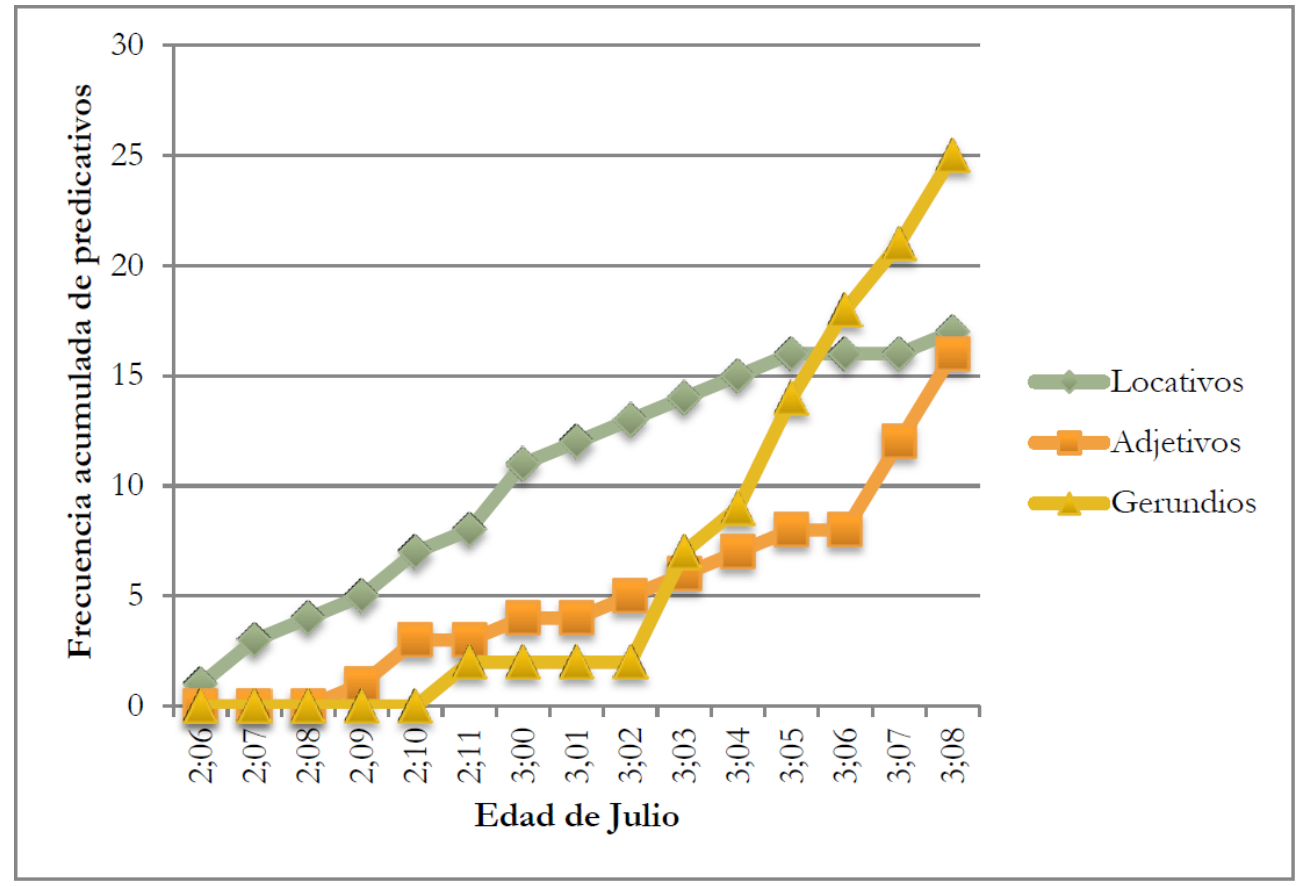

Gráfico 4. Frecuencia acumulada de tipos de predicativos de Julio.

Este crecimiento paralelo entre las construcciones con adjetivo y las construcciones con gerundio no se confirma de una manera tan precisa en el caso de Julio, que se muestra en el Gráfico 4. Sin embargo, lo que parece claro es que el inicio del crecimiento de la perífrasis aspectual de Julio coincide con las otras dos niñas en que aparece en el habla infantil cuando la construcción locativa ha sido utilizada por un tiempo largo. Es necesario aclarar aquí que Julio es un niño que empezó a hablar tardíamente, por lo que debe considerarse su desarrollo lingüístico general como postergado, si se compara con el de Elia y Flor.

\section{Discusión}

La primera hipótesis deriva de la contradicción que se entabla entre los estudios que arrojan datos a favor de una correlación del input con respecto a determinados elementos lingüísticos de la adquisición infantil Saviciüté et al., 2018), y los resultados obtenidos por Mueller-Gathercole et al. (1999), en el caso particular de los gerundios. Para comprobar si existe influencia del HDN, se analizó tanto la correlación de las ocurrencias, como los primeros 25 tipos de perífrasis en el habla infantil con respecto al HDN. Los resultados comprueban mi hipótesis acerca de que sí existe una influencia en la adquisición, pero esta se limita a una etapa específica del desarrollo de la perífrasis 'estar' + gerundio. Según mis resultados, se puede afirmar que la influencia es significativa en una primera etapa de la adquisición del gerundio. Esto se afirma dado que se encuentra una correlación de las perífrasis de los niños con las 
ocurrencias del HDN solo en la base densa. Se propone aquí que esto se debe a que el niño identifica a esta construcción perifrástica en combinación con un verbo en específico (o un número limitado), gracias a los contextos de frecuencia alta de las ocurrencias en el HDN en la suma de contextos específicos concentrados (el corpus denso), antes de que se dispare su productividad sintáctica. Esta propuesta deriva también del hecho de que en Flor y Julio hay un solo tipo de construcción aspectual que se mantiene de dos o a cuatro meses, antes de que la curva muestre un aumento rápido. La correlación negativa de las perífrasis del HDN con las del habla infantil en contextos dispersos (corpus mensual) se da debido a que la suma de contextos específicos dispersos reduce la probabilidad de encontrar una correlación. En cambio, el corpus denso cubre un periodo de uso de la construcción en cuestión que permite detectar el uso focalizado en mayor diversidad de contextos, lo que ayuda a identificar una correlación en el habla infantil. Para ejemplificar esta propuesta digamos que la niña observa que la perífrasis permite describir, por ejemplo, que su tío, 'está pintando', porque pintar es algo que él hace frecuentemente y la mamá verbaliza frecuentemente este acto. Esto le permite a la niña producir esta construcción lingüística adquirida para verbalizar que su tío 'está pintando' (frecuencia alta de ocurrencia). Este sería el verbo o la producción única que permitiría el arranque, según se planteó en el marco teórico. La descripción del tío pintando, verbalizado en el HDN cuando realiza el acto 'está pintando' es un contexto focalizado que la niña detecta, pero se limita al uso de esa construcción hasta que puede generalizar esta construcción a otros contextos, p. ej. 'está comiendo', 'está jugando', 'está viendo'. Cuando esta generalización toma lugar, la variedad de gerundios que escucha del input (suma de los tipos), le ayudará a producir más. Esto se refleja en la correlación positiva con respecto a los primeros 25 tipos (Tabla 2). Mi hipótesis se comprueba, en tanto que la frecuencia de las ocurrencias en el HDN apoya el arranque de la perífrasis (producción única o limitada), en la que la memoria tiene un rol predominante (para una discusión al respecto ver Rojas Nieto, 2018) y la frecuencia de los tipos tiene una clara influencia sobre el periodo de desarrollo de la productividad. Por tanto se puede afirmar que la productividad de la perífrasis se nutre de los nuevos tipos que le provee el habla adulta.

La segunda hipótesis plantea que las producciones de la perífrasis aspectual son productivas desde el inicio, debido a que tienen una base semántica en común con las construcciones locativas con 'estar'. Para comprobar esta hipótesis se realizó un análisis de regresión lineal y exponencial, con base en la metodología planteada por Abbot-Smith y Behrens (2006). De acuerdo con los resultados obtenidos, las curvas de crecimiento de la producción de los primeros 25 tipos en la perífrasis aspectual en el habla infantil muestran un mejor ajuste lineal después de un largo periodo de uso nulo. Esto, a su vez, proyecta una curva de pendiente pronunciada, lo que me permite argumentar que la perífrasis 'estar' + gerundio tiene cierto grado de representación 
mental en el infante antes de su primera aparición en el habla infantil. A pesar de que localizamos una sola producción de base léxica para la perífrasis aspectual con gerundio, sostengo que es productiva desde el inicio, ya que el crecimiento de la curva muestra un mejor ajuste lineal después de un periodo de uso nulo o casi nulo. Si una construcción que comparte material léxico y funciones particulares con otra puede apoyar su adquisición (Abbot-Smith \& Behrens, 2006), este análisis apunta a que, en efecto, la construcción locativa con 'estar' apoya el rápido crecimiento de la perífrasis aspectual analizada aquí. Una vez adquirido el significado de la construcción 'estar' + LOC, el gerundio resulta una combinación ideal con el verbo 'estar' debido a su significado de fase/estadio.

Desde la base de un modelo conexionista, sostengo que los niños se basan en el templete de la construcción locativa [VBO.ESTAR ASOCIADO A CAMBIO $_{+}$ADV LOCATIVO (CAMBIANTE)] para construir la perífrasis aspectual. Tomando en cuenta el principio de este modelo, de que ambas sirven a propósitos comunicativos similares.

Por último, es necesario mencionar que en el marco teórico se planteó la posibilidad de que un cierto número de verbos en su acervo cognoscitivo le permiten al niño formar un patrón para generalizar la raíz verbal más el morfema -ndo del gerundio. Según un estudio de caso de Rojas Nieto (2011) de la niña Flor, el crecimiento de las flexiones verbales se da a partir de los 23 meses. En los resultados obtenidos para esta niña en particular podemos observar que el inicio del crecimiento de la perífrasis se da a los 26 meses, una vez que se ha vuelto más productiva en cuanto a su uso de flexiones verbales. Esto nos arroja evidencia sobre otro factor que juega un papel importante en una construcción que se adquiere de forma aparentemente repentina.

\section{CONCLUSIONES}

Los niños utilizan su primera perífrasis 'estar' + gerundio basados en contextos específicos de uso, p. ej. 'estát pintando' apoyados por la frecuencia de las ocurrencias en el HDN, pero por un tiempo corto no extienden este uso a otros verbos. Una vez que los niños han adquirido el significado del verbo 'estar', a través de la construcción locativa, y se han vuelto más productivos en el uso de sus flexiones verbales, serán capaces de generalizar su uso a otros contextos, con nuevos verbos que irán adquiriendo del HDN.

La relevancia de la investigación desarrollada consiste en la aportación de tres factores. i) Ser el primer estudio sobre adquisición del español en aportar conocimiento acerca de las construcciones adquiridas mediante el apoyo conceptual de otras, a través del método de análisis de correlación lineal y exponencial. ii) Ser el primer análisis de la influencia del input que utiliza una prueba de correlación de los primeros 25 tipos. iii) Aporta un acercamiento metodológico que permite considerar la influencia del HDN sobre el desarrollo lingüístico, atendiendo a la selección del 
corpus. En general, se puede decir que en esta investigación se analiza de una manera novedosa múltiples factores que influyen en la adquisición de una construcción particular.

\section{REFERENCIAS BIBLIOGRÁFICAS}

Abbot-Smith, K. \& Behrens, H. (2006). How known constructions influence the acquisition of other constructions: The german passive and future constructions. Cognitive Science, 30(6), 995-1026 [en línea]. Disponible en: https://doi.org/10.1207/s15516709cog0000_61

Ambridge, B. \& Blything, R. P. (2016). A connectionist model of the retreat from verb argument structure overgeneralization. Journal of Child Language, 43(6), 1245 1276. https://doi.org/10.1017/S0305000915000586

Barðdal, J. (2008). Productivity: Evidence From Case and Argument Structure in Icelandic. Amsterdam: John Benjamins.

Behrend, D., Harris, L. \& Cartwright, K. (1995). Morphological cues to verb meaning: Verb inflections and the initial mapping of verb meanings. Journal of Child Language, 22(1), 89-106 [en línea]. Disponible en: https://doi.org/https://doi.org/10.1017/S0305000900009648

Bittner, D., Dressler, W. U. \& Kilani-Schoch, M. (2003). Introduction. En M. Bittner, Dagmar, U. Dressler, U. Wolfgang \& M. Kilani-Schoch (Ed.), Development of Verb Inflection in First Language Acquisition (pp. vii-xxxvii). Berlin: Mouton de Gruyter.

Brown, R. (1973). A first language. The early stages. Cambridge, MA: Cambridge University Press.

Fernández Leborans, M. J. (1999). La predicación: Las oraciones copulativas. En I. Bosque \& V. Demonte (Eds.), Gramática Descriptiva de la lengua española. Las construcciones sintácticas fundamentales. Relaciones temporales, aspectuales y modales (pp. 2359-2523). Madrid: Espasa Calpe [en línea]. Disponible en: http://www.nebrija.com/revistalinguistica/files/articulosPDF/articulo_531f362074814.pdf

Goldberg, A. E. (1995). Constructions: A Construction Grammar approach to argument structure. Chicago: University of Chicago Press. Chicago: University of Chicago Press.

Harrison, C. (1992). Is there a natural order of acquisition in Spanish verb morphology? Estudios de Lingüistica Aplicada, 15 \& 16(10), 266-278. 
Hernández Pina, F. (1984). Teorías psico-sociolingüísticas y su aplicación a la adquisición del español como lengua materna. Madrid: Siglo XXI.

Hsu, N., Hadley, P. A. \& Rispoli, M. (2017). Diversity matters: Parent input predicts toddler verb production. Journal of Child Language, 44(1), 63-86 [en línea]. Disponible en: https://doi.org/10.1017/S0305000915000690

Jackson-Maldonado, D. \& Maldonado, R. (2002). Determinaciones semánticas de la flexión verbal en la adquisición del español. En C. Rojas Nieto \& L. de León Pasquel (Eds.), La adquisición de la lengua materna: Español, lenguas mayas, euskera. (pp. 165-196). Ciudad de México: UNAM-UAQ.

Jackson-Maldonado, D., Maldonado, R. \& Thal, D. J. (1998). Reflexive and middle markers in early child language acquisition: evidence from Mexican Spanish. First Language, 18(54), 403-429 [en línea]. Disponible en: https://doi.org/10.1177/014272379801805407

Li, P. \& Bowerman, M. (1998). The acquisition of lexical and grammatical aspect in Chinese. First Language, 18(54), 311-350 [en línea]. Disponible en: https://doi.org/10.1177/014272379801805404

MacWhinney, B. \& O'Grady, W. (Eds.) (2015). The Handbook of Language Emergence. West Sussex: Wiley-Blackwell.

Mueller-Gathercole, V. C., Sebastián, E. \& Soto, P. (1999). The early acquisition of Spanish verbal morphology: Across-the-board or piecemeal knowledge? International Journal of Bilingualism, 3, 133-184 [en línea]. Disponible en: https://doi.org/10.1177/13670069990030020401

Ponce, X. T. (2008). La adquisición temprana de los verbos ser y estar en español. Tesis de magíster, Universidad Nacional Autónoma de México, México.

Rojas Nieto, C. (2007). La base de datos ETAL. Origen, descripción y metas de un proyecto. En Jornadas Filológicas 2005 (pp. 571-601). Ciudad de México: Instituto de Investigaciones Filológicas-UNAM.

Rojas Nieto, C. (2011). Developing first contrasts in Spanish verb inflection. En I. Arnon \& E. V. Clark (Eds.), Experience, Variation and Generalization (pp. 53-72). Ámsterdam: John Benjamins.

Rojas Nieto, C. (2018). Afinidades y diferencias individuales en el desarrollo temprano de la flexión verbal. En C. Rojas Nieto \& V. Oropeza (Eds.), Diferencias individuales en la adquisición del lenguaje. Factores lingüisticos, cognitivos, socioambientales (pp. 35-80). Ciudad de México: Universidad Autónoma de la Ciudad de México. 
Rojas Nieto, C. (en prensa). Constructional grounding in emerging constructions in Spanish acquisition complexity: Early. En C. C. Z. Estrada \& A. Álvarez (Ed.), Diachronic aspects of syntactic complexity: Inter and intra-typological diversity (pp. 1-31). Ámsterdam: John Benjamins.

Romberg, A. R. \& Saffran, J. R. (2011). Statistical learning and language acquisition. Wiley Interdisciplinary Reviews. Cognitive Science, 1(6), 906-914 [en línea]. Disponible en: https://doi.org/10.1002/wcs.78.Statistical

Ruhland, R. \& Geert, P. (1998). Jumping into syntax: Transitions in the development of closed class words. British Journal of Developmental Psychology, 16(1), $65-95$ [en línea]. Disponible en: https://doi.org/10.1111/j.2044-835X.1998.tb00750.x

Saviciüté, E., Ambridge, B. \& Pine, J. M. (2018). The roles of word-form frequency and phonological neighbourhood density in the acquisition of Lithuanian noun morphology. Journal of Child Language, 45, 641-672.

Shirai, Y. (2014). Semantic bias and morphological regularity in the acquisition of tense-aspect morphology: What is the relation? Linguistics [en línea]. Disponible en: https://doi.org/10.1515/LING.2010.005

Shirai, Y., Slobin, I. D. \& Weist, E. R. (2000). Introduction: The acquisition of tenseaspect morphology. First Language, 18(54), 245-253 [en línea]. Disponible en: https:/ / doi.org/10.1177/014272370002006005

Siegelman, N., Bogaerts, L., Elazar, A., Arciuli, J. \& Frost, R. (2018). Linguistic entrenchment: Prior knowledge impacts statistical learning performance. Cognition, 177(April), 198-213 [en línea]. Disponible en: https://doi.org/10.1016/j.cognition.2018.04.011

Soto, G. \& Castro, C. (2010). Una caracterización funcional de estar + gerundio como aspecto de fase: Progresividad, dinamicidad y lectura de caso (token). Revista de Lingüistica Teórica y Aplicada, 48(2), 93-113.

Stoll, S. (1988). The role of Aktionsart in the acquisition of Russian. First Language, 18, 351-377.

Tomasello, M. (1992). First verbs: A case study of early grammatical development. Cambridge: Cambridge University Press.

\section{NOTA}

${ }^{1}$ Nos referimos al término 'productividad sintáctica' que surge desde la gramática generativa y se define como la capacidad del hablante de producir y comprender oraciones que nunca ha escuchado antes (Barðdal, 2008). 